\title{
Integrazione pubblico-privato su metodologie e strumenti di controllo gestionale
}

\author{
Luciano Marchi*
}

Le finalità della rivista Management Control sono quelle di sviluppare il dibattito scientifico ed il confronto tra mondo accademico e operatori aziendali per l'approfondimento delle tematiche di pianificazione, informazione e controllo, con il ricorso ad un approccio integrato di analisi.

Il nuovo approccio di analisi, in particolare, si riferisce alla necessaria integrazione dei sistemi operativi con quelli informativo-contabili, della struttura organizzativa con la tecnologia informatica, dell'analisi revisionale con quella prospettica, anche al fine di raccordare i sistemi di misurazione con quelli di governo aziendale, nel riferimento indistinto ad aziende pubbliche e private, profit e non-profit.

Nel presente editoriale l'attenzione è rivolta all'ultimo dei punti sopra indicati, un possibile e auspicabile approccio di analisi integrato pubblicoprivato, per indagare sulle uniformità e difformità presenti nei relativi sistemi di governo e controllo gestionale.

La riflessione parte necessariamente dalle finalità che tutte le aziende pubbliche e private, profit e non-profit - dovrebbero perseguire: il soddisfacimento dei bisogni umani e un equilibrio economico a valere nel tempo.

Con riferimento alla prima finalità, anche alla luce dei cambiamenti in atto nei sistemi economici, nella prospettiva della cosiddetta economicosociale, si assiste ad un sempre maggiore avvicinamento tra aziende pubbliche e private:

- nelle aziende pubbliche, la finalità prioritaria del soddisfacimento dei bisogni umani è direttamente perseguita collegando sempre più la rendicontazione sociale al controllo interno gestionale;

\footnotetext{
*Università di Pisa; e-mail: Imarchi@ec.unipi.it
} 
- nelle aziende private, un sempre più diffuso controllo sociale spinge anche le aziende con finalità "profit" a perseguire in vario modo il consenso della comunità sociale ed il soddisfacimento, indiretto, dei bisogni umani.

Questa diffusa esigenza di tutela "sociale" si è affermata anche nella comunicazione esterna, sia quella obbligatoria che quella volontaria. Tale comunicazione esterna si amplia infatti per esprimere sia la consistenza patrimoniale e gli andamenti economico-finanziari delle aziende, sia la corretta amministrazione, il sistema dei valori aziendali e, più in particolare, l'efficace funzionamento dei loro sistemi di controllo interno.

Per quanto riguarda la seconda finalità, l'equilibrio economico a valere nel tempo, l'efficacia e l'efficienza che esso tipicamente contiene con riferimento alle aziende private si sono diffusamente affermate anche nelle aziende pubbliche.

Ritornando ai sistemi di governo e di controllo aziendale, le metodologie e gli strumenti sviluppati nell'ambito delle aziende private sono sempre più diffusi nelle aziende pubbliche.

Con riferimento al presente numero della rivista, i primi due articoli sono riferiti a tematiche di pianificazione e controllo applicati ad aziende private, ma applicabili anche ad aziende pubbliche, il terzo e quarto articolo trattano tematiche di pianificazione e controllo, con applicazioni relative ad aziende pubbliche, il quinto contributo è genericamente riferito alla valutazione dei sistemi di controllo interno, senza specificazioni privato-pubblico.

Nel primo articolo proposto da Antonio Leotta si discute sul ruolo del controllo nello sviluppo di nuovi prodotti. Nella parte teorica si confrontano gli studi sui processi di sviluppo con quelli sul controllo di tali processi, con particolare riferimento alla teoria della strutturazione di Giddens. Nella parte applicativa viene presentato un case study sul processo di sviluppo di una multinazionale operante nel settore dei semiconduttori.

Quale possibile estensione alla realtà delle aziende pubbliche?

La principale area di interesse per le aziende pubbliche è quella che, a partire dalla natura composita dei processi di sviluppo, produttiva e creativa, riflette il ruolo strategico della conoscenza come risorsa da sostenere per supportare un equilibrio economico durevole. Vale per le aziende private, ma vale, nello stesso modo, per le aziende pubbliche, in quanto si considerano le modalità con cui, nei sistemi di controllo, certi valori influenzano le interazioni sociali e, mediante queste ultime, si riproducono nel tempo.

Il secondo articolo, di Andrea Guerrini, tratta dei sistemi di analisi degli scostamenti per la valutazione delle performance realizzate rispetto a quelle programmate. L'analisi è svolta a partire dai classici modelli di analisi degli 
scostamenti applicati ai ricavi di vendita ed ai margini reddituali delle aziende di produzione. Il contributo dall'autore al dibattito scientifico sull'argomento si realizza su tre aspetti: l'impiego di formule analitiche in grado di articolare maggiormente la varianza di mix; l'applicazione dell'analisi degli scostamenti anche ai valori patrimoniali; la possibilità di aggregare sottoscostamenti riferiti a valori distinti per natura, economici o patrimoniali, ma riferiti ad una stessa dimensione di analisi al fine di poter valutare l'economicità dei corsi d'azione intrapresi.

Anche in questo caso la domanda che si pone è relativa alle possibili estensioni alle aziende pubbliche delle ricerche sull'analisi degli scostamenti. Si possono ipotizzare, al riguardo, approfondimenti con riferimento alla dimensione sia economica che finanziaria.

Il terzo articolo, di Luisa Pulejo, considera il profilo di genere nella programmazione delle aziende pubbliche territoriali. L'obiettivo del lavoro è quello di fornire alcuni spunti di riflessione su un cambiamento culturale "performance oriented", nella elaborazione delle strategie e la valutazione delle scelte effettuate, dei risultati attesi e di quelli realizzati, che parte dal sistema dei documenti programmatici di bilancio.

Si sostiene, al riguardo, l'opportunità di realizzare un processo partecipativo e trasparente volto a conoscere i bisogni e le aspettative che i cittadini esprimono in relazione alle loro diversità di "genere", dove il genere viene inteso come "categoria sociale", ma viene analizzato anche nella sua "valenza economica".

È proprio quest'ultimo punto, la valenza economica del profilo di genere, che potrebbe utilmente essere utilizzato per approfondire le ricerche di "genere" con un approccio integrato pubblico-privato.

Il quarto articolo, a cura di Elena Gori e Silvia Fissi, affronta la tematica della contabilità analitica come presupposto per la determinazione degli standard di costo negli enti locali e si pongono la seguente domanda di ricerca: è la contabilità analitica sufficiente?

A partire dalla recente riforma federalistica che, dal punto di vista contabile, si basa sulla determinazione degli standard di costo, risulta che solo il $27 \%$ degli enti locali italiani abbia adottato un sistema di contabilità analitica che, almeno in linea teorica, dovrebbe essere in grado di determinare gli standard.

Le conclusioni a cui pervengono le autrici, attraverso un case study, sono che l'adozione di un sistema di contabilità analitica per centri di costo sia presupposto necessario ma non sufficiente per determinare realmente $\mathrm{i}$ costi standard dei servizi erogati. È necessaria, infatti, una maturità applica- 
tiva della contabilità analitica per centri di costo o per attività che, ad oggi, la maggior parte degli enti locali non ha sviluppato.

Dall'indagine di Gori e Fissi scaturiscono alcune riflessioni ed una ulteriore domanda di ricerca: se le condizioni gestionali e l'ambiente di controllo delle aziende - sia private che pubbliche - siano in grado di determinare lo sviluppo della indicata maturità applicativa.

Più in generale, coerentemente con le finalità di indagine definite per la rivista Management Control, un tema di grande interesse, sia per le aziende private che per le aziende pubbliche, è quello della valutazione dei sistemi di controllo interno.

È questo l'oggetto del quinto articolo proposto da Rita Lamboglia che, affrontando il tema della valutazione, con un'analisi della letteratura italiana ed internazionale degli ultimi venti anni, approfondisce particolarmente la componente soggettiva del sistema di controllo identificata con il termine "ambiente di controllo".

L'analisi della letteratura è stata necessariamente delimitata nel riferimento ad un insieme di riviste e di editori con una ricerca effettuata attraverso parole chiave, senza una specifica considerazione delle aziende pubbliche, che potrebbero essere utilmente indagate in un successivo studio comparativo pubblico-privato centrato sull'ambiente di controllo.

In conclusione, riteniamo, che alcuni principi generali debbano essere rivisti, anche in rapporto all'evoluzione dei sistemi di controllo in termini di integrazione interno-esterno, della dimensione economica e di quella sociale. Nelle aziende private il controllo si pone sempre più l'obiettivo di soddisfare le esigenze di conoscenza esterne e realizzare nuovi modelli di controllo delle relazioni cooperative con clienti, fornitori, finanziatori, lavoratori e, più in generale, con la comunità sociale, dunque sistemi di governance economico-sociale.

Nelle aziende pubbliche, d'altra parte, il controllo è sempre più rivolto a soddisfare obiettivi di economicità intesa non solo in senso giuridicoformale (economicità nell'acquisizione delle risorse) ma anche in senso sostanziale (integrazione degli obiettivi di efficace ed efficiente governo della gestione). 